\title{
Middle Miocene Benthic Foraminifera from Wadi Kaam area, Northwestern Libya: Systematics and biostratigraphy
}

\author{
Sherif M. El Baz*, Mohamed A. Ahmed, Abd-Alrahman A., Embaby, Ahmed Al Furjany \\ Damietta University, Faculty of Science, Geology Department, New Damietta, Egypt.
}

Received: 12 December 2016 /Accepted: 5 March 2017

*Corresponding author: Sherif772003@du.edu.eg

\begin{abstract}
The Middle Miocene section exposed at Wadi Kaam area, northwestern Libya was carefully studied. Detailed investigation of the foraminiferal content led to the recognition of 27 benthic foraminiferal species belonging to 15 genera and 13 families. They are moderately preserved. The absence of the index planktonic foraminifera doesn't enable the recognition of any planktonic biozone within Al Khums Formation. The studied Miocene succession includes one biostratigraphic zone based on the vertical distribution of the larger benthic foraminifera Borelis melo melo. The studied sequence was characterized by a fairly common occurrence of benthic foraminiferal species living in a shallow neritic environment.
\end{abstract}

Keywords: Foraminifera, Middle Miocene, Taxonomy, Biostratigraphy, Libya.

\section{Introduction}

This study is concerned with the Middle Miocene benthic foraminifera from exposed section at Wadi Kaam area in the northwestern Libya (Fig. 1). It is directed to their taxonomy and biostratigraphy. Few studies have been published on the Miocene foraminifera of Libya. Significant papers are those by Berggren (1967), Barr \& Weegar (1972), Szczechura \& AbdElshafy (1988), Sherif (1991), Abdulsamad \& Barbieri (1999), Imam (1999a), Hamad (2013), Abdulsamad \& El Zanati (2013).

\section{Material and Methods}

In the present study, a total of 30 samples were carefully collected and detailed studied. The samples were prepared to release the foraminiferal content. All the identified foraminifera were photographed by the Scanning Electron Microscope. The photographs were arranged in two plates.

\section{Lithostratigraphy}

In the study area, the Middle Miocene succession is mainly comprised of the $\mathrm{Al}$ Khums Formation.

It was described and established by Mann (1975) for the Middle Miocene sequence 
exposed in the vicinity of $\mathrm{Al}$ Khums. It can be locally subdivided by Salem \& Spreng (1980) into two informal members, from base to top: An Naggazah Member and Ras Al Mannubiyah Member. In the present study, An Naggazah Member composed mainly of reefal limestone at the base followed by white, massive, and fossiliferous limestone, with pelecypods, gastropods, bryozoan fragments, foraminifera and ostracods. It reaches about 9 m (Fig. 2). Ras Al Mannubiyah Member overlies conformably An Naggazah Member and attains a thickness of $6 \mathrm{~m}$. It consists of white creamy limestone with rare ostracods and foraminifera. Borelis melo melo was considered as a marker for the Middle Miocene beds (Barr \& Weegar, 1972; Imam, 1999 a, Hamad, 2013). It is clear that the presence of Borelis melo melo in the present study gives a strong support that the Al Khums Formation is of Middle Miocene (Langhian to Early Serravallian) age.

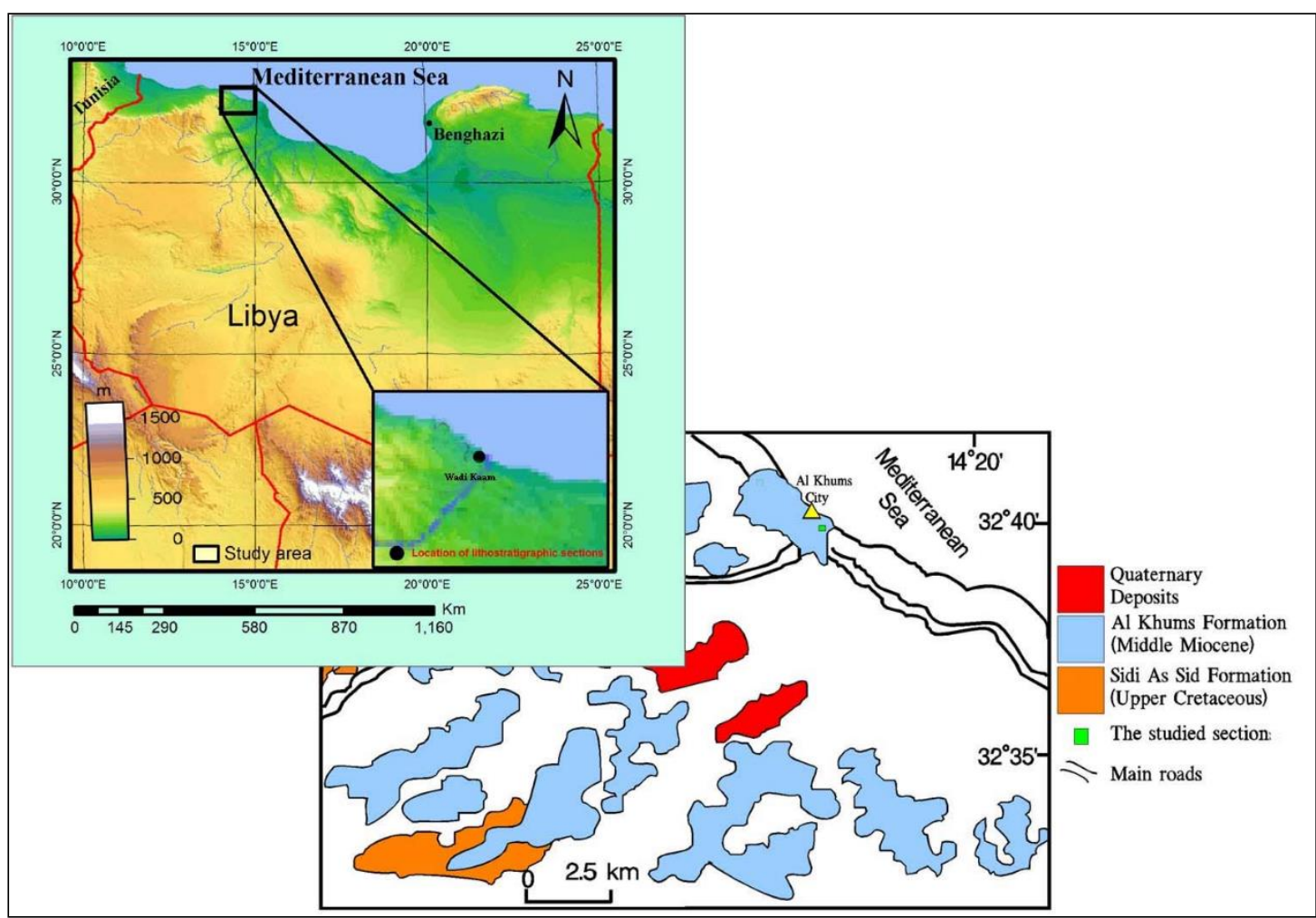

Fig.1. Geologic map of the study area (modified after, Hamad, 2013).

\section{Systematic Paleontology}

Detailed investigation of the foraminiferal content led to the recognition of 27 species belonging to 15 genera and 13 families. The recorded species follow the classification of Loeblich \& Tappan (1988). They are illustrated in two plates and their stratigraphic ranges are provided in Fig. 2. Order: Foraminiferida Eichwald, 1830

Suborder: Miliolina Delag \& Herouard, 1896

Superfamily: Miliolacea Ehrenberg, 1839

Family: Spiroloculinidae Wiesner, 1920

Genus: Spiroloculina d'Orbigny, 1826

Spiroloculina communis Cushman \& Todd, 1884, (P1. 1, Fig. 1)
1963 Spiroloculina cf. S. communis Cushman \& Todd -Souaya, pl. 58, fig. 5. Family: Hauerinidae Schwarger, 1876 Subfamily: Hauerininae Schwager, 1876 Genus: Quinquloculina d'Orbigny, 1826 Quinquloculina laevigata d'Orbigny, 1826 , (Pl. 1, Fig. 2)

1826 Quinqueloculina laevigata d'Orbigny, p. 301, no. 6. Quinqueloculina cuvieriana d'Orbigny, 1839, (P1. 1, Fig. 3)

2013 Quinqueloculina cuvieriana d'Orbigny-Hewaidy et al., pl. 1, fig. 1.

Quinqueloculina seminula (Linnaeus, 1758), (P1. 1, Fig. 4)

2013 Quinqueloculina seminulua (Linnaeus)-Hewaidy et al., pl. 1, fig. 3.

Quinquloculina vulgaris d'Orbigny, 1826, (Pl. 1, Fig. 5) 
1826 Quinquloculina vulgaris d'Orbigny, $\mathrm{p}$. 302, no. 33.

Subfamily: Miliolinellinae Vella, 1957

Genus: Triloculina d'Orbigny, 1826

Triloculina gibba d'Orbigny, 1826 , (P1. 1, Fig. 6)

1974 Triloculina gibba d'orbignyLuczkowska, p. 134, pl. 23, figs. 2 a-c.

Triloculina inflata d'Orbigny 1846, (Pl. 1, Fig. 7)

1991 Triloculina inflata d' Orbigny Sherif, pl.2, figs. 2a,b.

Triloculina tricarinata d'Orbigny, 1826, (Pl. 1, Fig. 8)

1826 Triloculina tricarinata d'Orbigny, Annals des sci. Naturelles, p.209, v. 7, model no. 94.

Triloculina trigonula (Lamark, 1804), (Pl. 1, Fig. 9)

1991 Triloculina trigonula (Lamark) Sherif, pl.2, figs. 53a,b.

Family: Alveolinellidae Cushman, 1927

Genus: Borelis Montfort, 1808

Borelis melo melo (Fichtel \& Moll, 1798),

(Pl. 1, Fig. 10),

1798 Nautilus melo Fichtel \& Moll, p. 123, pl. 24, figs. a-h.

Suborder: Lagenina Delage \& Herouard, 1896

Superfamily: Nodosariacea Ehrenberg, 1838

Family: Vaginulinidae Reuss, 1860

Subfamily: Lenticulininae Chapman, Parr \& Collins, 1934

Genus: Lenticulina Lamarck, 1804

Lenticulina inornatus (d'Orbigny, 1846), (Pl. 1, Fig. 11)

1846 Robulina inornata d 'Orbigny, p. 102, pl. 24, figs. 25-26

Family: Polymorphinidae d'Orbigny, 1839

Genus: Guttulia d'Orbigny, 1839

Guttulina communis (d'Orbigny, 1829), (Pl.

1, Fig. 12)

1829 Polymorphina communis d'Orbigny, p. 150 , pl. 9

Suborder: Rotaliina Delage \& Herouard, 1896

Superfamily: Bolivinacea Glaessner, 1937

Family: Bolivinidae Glaessner, 1937

Genus:Bolivina d'Orbigny, 1839

Bolinina arta Macfadyen, 1931, (Pl. 1, Fig.

13)

1931 Bolivina arta Macfadyen, p. 58, pl. 4, fig. 2 la-b.

Bolivina dilatata Reuss, 1850, (Pl. 1, Fig. 14)
1931 Bolivina dilatata Reuss - Macfadyen, p. 57, pl. 2, figs. la-b.

Family: Uvigerinidae Jones, 1875

Subfamily: Uvigerininae Haeckel, 1894

Genus:Uvigerina d'Orbigny, 1826

Uvigerina semiornata d'Orbigny, 1826, (Pl.

2, Fig. 1)

1953 Uvigerina semiornata d'Orbigny -

Papp \&Turnovsky, p. 128, pl. 5, figs. C1-3.

Superfamily: Orbitoidacea Schwager, 1876

Family: Amphisteginidae Cushman, 1927

Genus: Amphistegina d'Orbigny, 1826

Amphistegina lessonii (d' Orbigny, 1843),

(Pl. 2, Fig. 2)

1991 Amphistegina lessonii (d' Orbigny) Sherif, pl. 4, figs. 7a,b.

Superfamily: Nonionacea Schultze, 1854

Family: Nonionidae Schultze, 1854

Subfamily: Nonioninae Schultze, 1854

Nonion boueanus d'Orbigny, 1846, (Pl. 2, Fig. 3)

1991 Nonion boueanus d'Orbigny-Sherif., pl. 5, figs. 7a,b.

Family: Heterolepidae Gonzales-Donoso, 1969

Genus : Heterolepa Franzenau 1884

Heterolepa dutempli (d'Orbigny, 1846),

(Pl. 2, Figs. 4, 5)

1846 Rotalinad dutempl d 'Orbigny, p. 157, pl. 8, figs. 19-21.

Family: Gavelinellidae Hofker, 1956

Subfamily: Gavelinellina Hofker, 1956

Genus: Hanzawaia Asano, 1944

Hanzawaia boueana (d'Orbigny, 1846), (Pl. 2, Fig. 6)

1965 Cibicides boueanus (d'orbigny)Souaya, pl. 3, figs. 1a,b.

Family: Rotaliidae Ehrenberg, 1839

Subfamily: Pararotaliinae Reiss, 1963

Genus: Pararotalia Le Calvez, 1949

Pararotalia armata (d'Orbigny, 1826), (Pl.

2, Fig. 7)

1988 Pararotalia armata (d'Orbigny)Szczechura \& Abd-Elshafy, pl. 12, figs. 1, 2.

Pararotalia mexicana (Nuttall, 1928), (Pl.

2, Fig. 8)

1928 Rotalia mexicana (Nuttall), pl. 50, figs. 6-7.

Subfamily: Ammoninae Saidova, 1981

Genus: Ammonia Brunnich, 1771

Ammonia beccarii (Linné, 1758), (Pl. 2, Fig. 9)

1758 Nautilus beccarii Linné, p. 710.

Ammonia parkinsoniana (d'Orbigny, 1839), (Pl. 2, Fig. 10) 
1839 Rosalina parkinsoniana d'Orbigny, $\mathrm{p}$. 99, pl. 4, figs. 25-27.

Family: Elphidiidae Galloway, 1933

Subfamily: Elphidiinae Galloway, 1933

Genus: Elphidium Montfort, 1808

Elphidium advenum (Cushman, 1922), (Pl.

2, Fig. 11)

1939 Elphidium advenum (Cushman)Cushman, pl. 16, figs. 31-35.

Elphidium crispum (Linee 1758), (Pl. 2, Fig. 12)

1965 Elphidium crispum (Lineé)- Souaya, pl. 3, fig. 13.

Elphidium macellum (Fichtell \& Moll, 7981), (Pl. 2, Fig. 13)

1965 Elphidium macellum (Fichtell \& Moll) - Souaya, pl.3, fig. 10.

Elphidium minutum (Reuss, 1864), (Pl. 2, Fig. 14)

1965 Elphidium cf minutum (Reuss) Souaya, pl. 3, fig. 12.

\section{Biostratigraphy}

In the present study, the absence of the index planktonic foraminifera doesn't enable the recognition of any planktonic biozone. The studied Miocene section include one biostratigraphic zone based on the vertical distribution of Borelis melo melo.

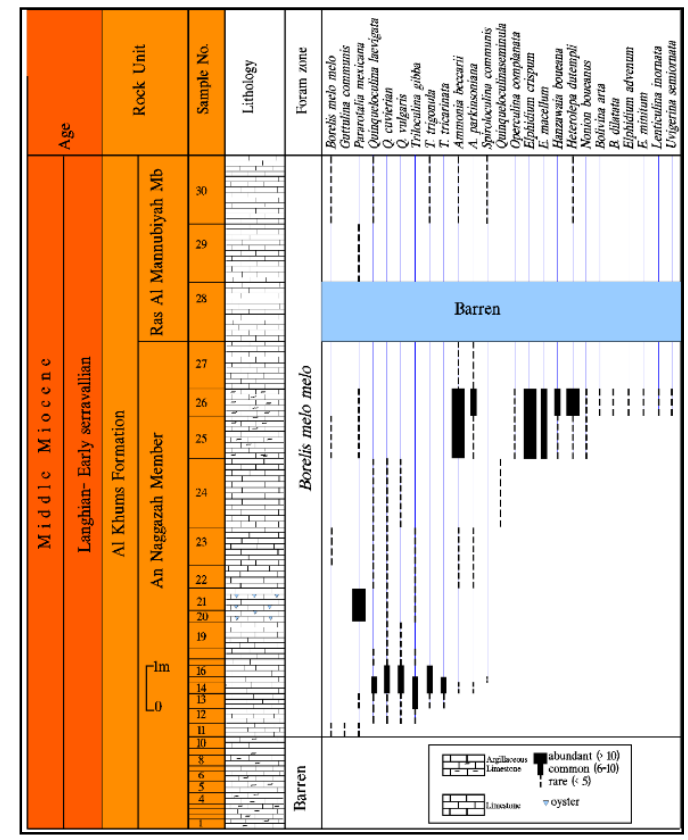

Fig.2. Stratigraphic range charts of the recorded foraminifera.

\section{Borelis melo melo Biozone}

This zone is defined in the present work by the total range of the nominated taxon. It covers the whole Al Khums Formation. The associated foraminifera include Pararotalia mexicana, Quinqueloculina spp, Triloculina spp., Elphidium crispum, Heterolepa dutempli and Ammonia beccarii. The biostratigraphic importance of this taxon as Middle Miocene index species have been discussed by many authors. Souaya (1963) recorded this species from the Middle Miocene succession in the Red Sea, Egypt. Sherif (1991) recorded it from Al Khums Formation in Al Khums area, NW Libya. $\operatorname{Imam}(1999 \mathrm{a}, \mathrm{b})$ also recorded this biozone in the Middle Miocene Al Jaghboub Formation in Al Bardia area, NE Libya. Hamad (2013) defined this zone from the Middle Miocene Al Khums Formation. Abdulsamad \& Zanati (2013) identified this species from the Soluq area, NE Libya. This biozone could be correlated with the Praeorbulina glomerosa, Orbulina suturalis and Orbulina universa planktonic foraminiferal Zones.

Due to the abovementioned discussion, the presence of Borelis melo melo in the present study gives a strong support that $\mathrm{Al}$ Khums Formation is of Middle Miocene age (Langhian-Early Serravallian).

\section{Conclusions:}

Detailed investigation of the foraminiferal content from the stratigraphic section that exposed at Wadi Kaam area led to the recognition of 27 benthic foraminiferal species belonging to 15 genera and 13 families. Based on the vertical distribution of Borelis melo melo, one biostratigraphic zone has been recognized. The recorded benthic foraminifera indicated shallow neritic environment. The co-occurrence of corals, large oysters and algae supports shallow marine shelf environments for the deposition of Al Khums Formation.

\section{Plate 1}

Scale bar $=100 \mu \mathrm{m}$, except for $7,13,14=$ $50 \mu \mathrm{m}$. 
Fig.1: Spiroloculina communis Cushman \& Todd, sample no. 30.

Fig.2: Quinquloculina laevigata d'Orbigny, sample no. 30.

Fig.3: Q. cuvieriana d'Orbigny, sample no. 24.

Fig.4: $Q$. seminula (Linnaeus), sample no. 24.

Fig.5: $Q$. vulgaris d'Orbigny, sample no. 15.

Fig.6: Triloculina gibba d'Orbigny, sample no. 23 .

Fig.7: T. inflata d'Orbigny, sample no. 24.

Fig.8: T. tricarinata d'Orbigny, sample no. 30.

Fig.9: T. trigonula (Lamark), sample no. 30.

Fig.10: Borelis melo melo (Fichtel \& Moll), sample no. 25.

Fig.11: Lenticulina inornatus (d'Orbigny), sample no. 26.

Fig.12: Guttulina communis (d'Orbigny), sample no. 11.

Fig.13: Bolinina arta Macfadyen, sample no. 26 .

Fig.14: B. dilatata Reuss, sample no. 26.

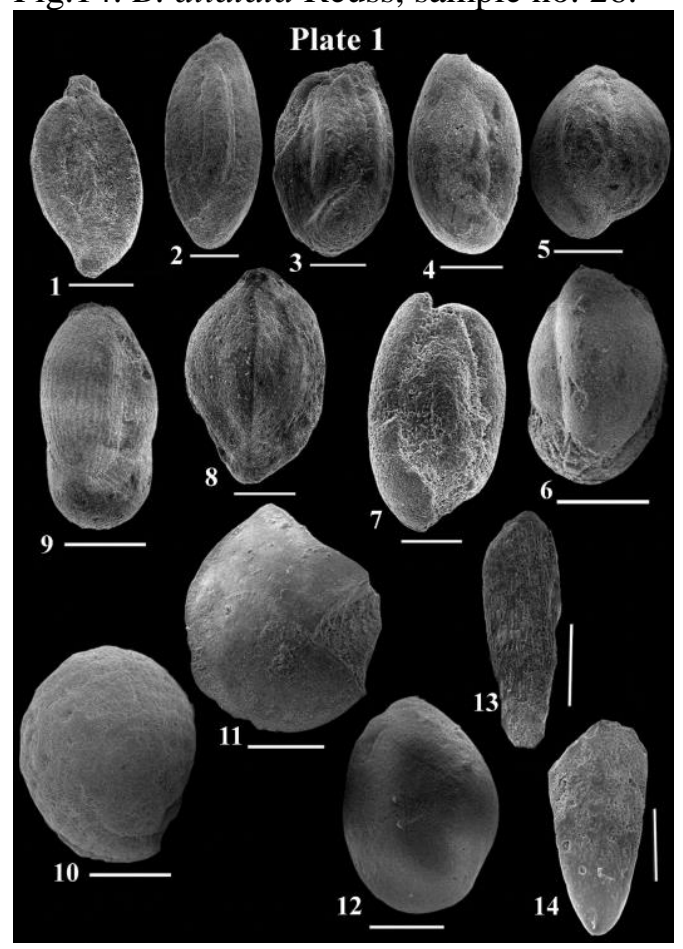

Plate 2

Scale bar $=100 \mu \mathrm{m}$, except for $3,6,8,9$, $10,14=50 \mu \mathrm{m}$.

Fig.1:Uvigerina semiornata d'Orbigny, sample no. 26.

Fig.2: Amphistegina lessonii (d' Orbigny), sample no. 24
Fig.3: Nonion boueanus d'Orbigny, sample no. 26.

Figs.4, 5: Heterolepa dutempli (d'Orbigny), sample no. 25.

Fig.6:Hanzawaia boueana (d'Orbigny), sample no. 25.

Fig.7: Pararotalia armata (d' Orbigny), sample no. 29.

Fig.8: Pararotalia mexicana (Nuttall), sample no. 21.

Fig.9: Ammonia beccarii (Linné), sample no. 27.

Fig.10: Ammonia parkinsoniana (d'Orbigny), sample no. 27.

Fig.11: Elphidium advenum (Cushman), sample no. 26.

Fig.12: E. crispum (Linee), sample no. 26.

Fig.13: E. macellum (Fichtell \& Moll), sample no. 26.

Fig.14: E. minutum (Reuss), sample no. 26.

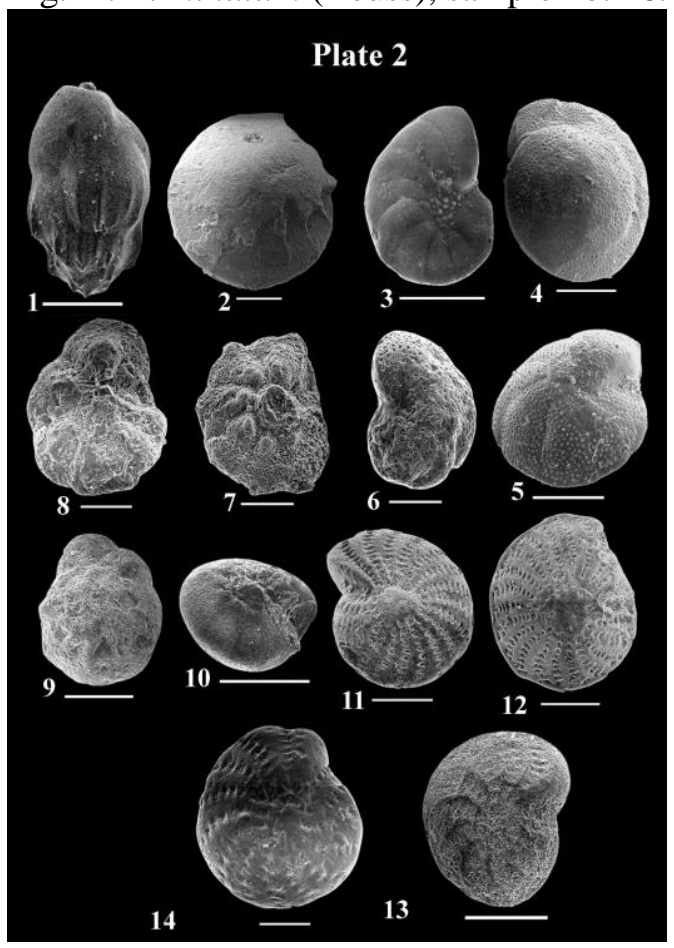

\section{References}

Abdulsamad EO, Barbieri R (1999) Foraminiferal distribution and palaeoecological interpretation of the Eocene-Miocene carbonates at Al Jabal Al Akhdar (NE Libya). Jour. Micropalaeontol, 18: 45-65.

Abdulsamad EO, El Zanati SM (2013) Miocene benthic foraminifera from the Soluq area, NE Libya: biostratigraphy and environmental significance. Jour. Mediterran. Ear.Sci. 5, 245-256. 
Barr FT \& Weegar AA (1972) Stratigraphic nomenclature of Sirte Basin, Libya. Petroleum Exploration Society : 179.

BerggrenWA (1967) Biostratigraphy and planktonic foraminiferal zonation of the Tertiary system of Sirte Basin of Libya. North African. Proceeding of the 1st international Conference on Planktonic Microfossils, Geneva: 104-120.

Hamad MM (2013) Biostratigraphy and paleoecology of the Miocene sequence along the stretch of Qabilt ash Shurfah to Wadi Zaqlum sections, Sirte Basin, Libya. Australian Journal of Basic and Applied Sciences, 7(10): 513-531.

Imam MM (1999a) Middle Miocene sequence in the area between Wadi Al Zeitun and Wadi Al Rahib, Al Bardia area, northeast Libya. Journal of African Earth Sciences, 28(3): 619-639.

Loeblich AR, Tappan H (1988) Foraminiferal genera and their classification. - Bd.I:I-X: 1-970; Bd. II:I-VII: 1-212, 874 pl., (Van Nostrand Reinholked Company); New York.
Mann K (1975). Geological Map of Libya. 1:250.000, Al Khums sheet (NH $33-14$ ). Explanatory Booklet. Industrial Research Center, Tripoli, 88.

Salem MJ, Spreng AC (1980) Middle Miocene stratigraphy, Al Khums area, NW Libya. In: The Geology of Libya (Eds. Salem, M.J. and Busrewil, M. T.) Fac. Science, Univ. of Al Fatah, Tripoli, I: 97-116.

Sherif KAT (1991) Biostratigraphy of the Miocene in Al Khums area, NW Libya. In: The Geology of Libya (Eds: Salem, M. J., Hammouda, O. S. and Eliagoubi, B. A.) Faculty of Sciences, University of Al Fatah, Tripoli, IV: 1421-1456.

Souaya FJ (1963) On the foraminiferao f Gebel Gharra( Cairo-Suez Road) and some other Miocene samples. Jour. Paleontol. 37(2): 433-457.

Szczechura J, Abd-Elshafy (1988). Ostracodes and Foraminifera from the ?Middle Miocene of the western coast of the gulf of Suez, Egypt. Acta Palaeontologica polonica.: 33:273-342.

عنوان البحث: أحافير الفورامينيفرا فى الميوسين الأوسط من وادى كئام ، الخمس ، شمال غرب ليبيا

شريف الباز ، محمد أحمد ، عبد الرحمن امبابى ، أحمد الفرجانى

قسم لجيولوجيا ـ كلية العوم - جمعة دمياط

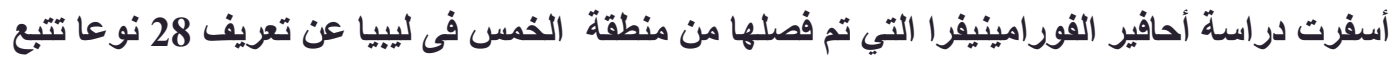

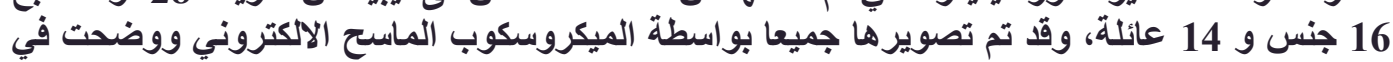

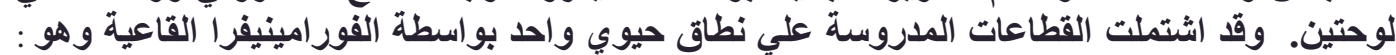

Borelis melo melo Biozone

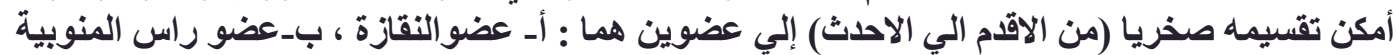

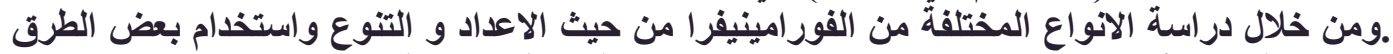

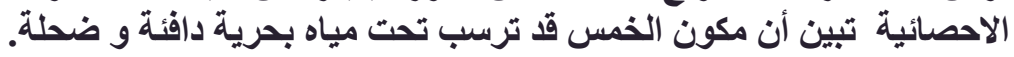

